\author{
Final Report \\ September 1985
}

Fawwaz T. Ulaby

Principal Investigator

This report was prepared for the Jet Propulsion Laboratory, California Institute of Technology, sponsored by the National Aeronautics and Space Administration.

Contract No. 956921 
M. C. Dobson and F. T. Ulaby

Electrical Engineering and Computer Science Department

University of Michigan

4072 East Engineering

Ann Arbor, Michigan 48109

D. R. Brunfeldt

Applied Microwave Corporation

Route 6, Box 205A

Lawrence, Kansas 66046

D. N. Held

Jet Propulsion Laboratory

California Institute of Technology

4800 Oak Grove Drive

Pasadena, California 91103

ABSTRACT

Data-takes on two ascending orbits of the Shuttle Imaging Radar- $B$ (SIR-B) over an agricultural test site in west-central Illinois were used to establish end-to-end transfer functions for conversion of the digital numbers on the 8-bit image to values of the radar backscattering coefficient $\sigma^{0}\left(\mathrm{~m}^{2} / \mathrm{m}^{2}\right)$ in dB. The transfer function for each data-take was def ined by the SIR-B response to an array of six calibrated point targets of known radar cross-section (transponders) and to a large number of area-extended targets also with known radar cross-section as measured by externally calibrated, truck-mounted scatterometers. The radar cross-section of each transponder at the SIR-B center frequency was measured on an antenna range as a function of local angle of incidence. 
Two truck-mounted scatterometers observed 20 to 80 agricultural fields daily at $1.6 \mathrm{GHz}$ with $\mathrm{HH}$ polarization and at azimuth viewing angles and incidence angles equivalent to those of the SIR-B.

The form of the transfer function is completely defined by the SIR-B receiver and the incoherent averaging procedure inorporated into production of the standard SIR-B image product. Assuming that the processing properly accounts for the antenna gain, all transfer function coefficient's are known except for the thermal noise power and a system "constant" which has been shown to vary as a function of uncommanded changes in the effective SIR-B transmit power. For each orbital pass, the SIR-B thermal noise was estimated from surface areas expected to yield specular reflection, and the system "constant" was determined for each area on the SIR-B image containing a target of known radar cross section. Both the point targets and the area-extended targets were found to yield nearly identical results with a mean difference of approximately $0.1 \mathrm{~dB}$. For a given date, the standard error of the estimate for the system "constant" as derived by this method is found to vary from $\pm 0.85 \mathrm{~dB}$ to \pm 1.35 dB. The interpass variance of the transfer functions was found to be related to the observed variance of the effective SIR-B transmit power. Application of the system transfer functions to SIR-B imagery permitted realization of science objectives by allowing comparison of multidate imagery on a common basis.

Five of the six transponders also operated as calibrated receivers. For each of six data-takes, two ascending and four descending, the receivers were distributed over an area extending approximately $20 \mathrm{~km}$ in both range and azimuth directions. For each SIR-B data-take, each receiver recorded the time history of a voltage proportional to the incident power density at the ground. The observed azimuth beam form appeared to be nominal with respect to specifications. Preliminary 
analysis of the range pattern, which could not be ascertained in a direct fashion with statistical confidence, indicates that the pattern may be nominal provided that the true antenna boresight is estimated to an accuracy of $+1-2^{0}$ via preliminary estimates of the STS ephemeris. Finally, the uncommanded loss of effective transmit power, which has been attributed to arcing, was found to average $7.1 \mathrm{~dB}$ and vary from pass-to-pass by approximately $3 \mathrm{~dB}$. 
In October of 1984, the Space Shuttle Challenger carried the Shuttle Imaging Radar (SIR-B) as part of the payload on the STS-4IG mission. The SIR-B instrument is an $\mathrm{HH}$-polarized L-band SAR $(1.28 \mathrm{GHz})$ capable of operating over incidence angles from about $15^{\circ}$ to $60^{\circ}$ relative to nadir. Although the mission was plagued by a series of hardware malfunctions, a vast quantity of data was acquired over both water and land surfaces. Much of this data was digitally recorded and transferred via TDRS-I for subsequent digital processing by the Jet Propulsion Laboratory. The digital SAR correlator incorporates both known and modeled system parameters in an attempt to produce digital image products free from system-related artifacts both in range and azimuth.

Since many of the science objectives of the mission required relative calibration of the SIR-B imagery in order to compare either multiangle or multisite observations, an important technical issue is the stability of the SIR-B instrument and the adequacy of the antenna patterns assumed in the SAR correlator. In addition, it is desireabie to evaluate existent means for providing end-to-end system transfer functions for the conversion of image digital number into units of radar backscattering coefficient $\sigma^{0}$ $\left(\mathrm{m}^{2} / \mathrm{m}^{2}\right)$.

In order to address these technical objectives, a test site was established in west-central Illinois at the intersect point of projected ascending and descending SIR-B coverage. Preceding the ascending data-takes, the Challenger generally conducted orbital alignment maneuvers so that the shuttle ephemerides could be precisely determined. During the mission, six digital data-takes over this site were obtained from three azimuth view angles and with local angles of incidence from $\approx 17^{\circ}$ to $59^{\circ}$ as given in Table 1. Of the six data-takes, digital imagery was produced for five swaths as lliustrated in Figure 1. 
On the ground, the test site comprised an irregular area of roughly $250 \mathrm{~km}^{2}$ intended to transect both ascending and descending image swaths. For science objectives related to the radar backscattering from vegetation canopies and underlying soil, this area was both extensively and intensively monitored on a daily basis for soil moisture and canopy biophysical conditions. For technical objectives, the site was instrumented with an array of six point targets and traversed on a daily basis by two L-band truck-mounted scatterometers.

The point targets, active radar calibrators (ARCs), function both as calibrated receivers and as transponders. Each ARC consists of two patch antennas (transmit and receive) connected by a detection circuit and a high gain RF amplifier [1]. As a receiver, interfacing the ARC with an instrumentation tape recorder permitted retrieval of the time-history of the SIR-B transmit power to yield azimuth cuts of the SIR-B antenna pattern. During each of the six SIR-B data-takes, the ARCs were distributed over the test site with spatial seperations of up to $20 \mathrm{~km}$ in range and/or azimuth. Subsequent processing of this data using the ARC transfer functions and estimates of the shuttie position and velocity to calculate the SIR-B power density at the ground also provided a means for examination of the cross-track (range) antenna pattern and comparison of pass-to-pass variability in the SIR-B transmitter's output power.

As transponders, the ARCs perform as point targets of known radar cross section. When properly deployed within an area characterized by a relatively low $\sigma^{\circ}$, the ARC is imaged as a bright target which serves as a calibration reference for establishment of the image transfer function. Although six ARCs were deployed within the test site during each data-take, misalignment of the SIR-B data window with respect to the test site on the descending orbits (Figure 1) restricted imaging of the ARCs to primarily the ascending data-takes (DT 49.2 and 97.2). 
An additional method for computation of the image transfer functions was provided by the observation of many area-extended targets by the truck-mounted sçatterometers. Two FM-CW scatterometers were used; both were operated at $1.6 \mathrm{GHz}$ with $\mathrm{HH}$ polarization. On a daily basis, each system observed 40 to 80 different agricultural fields distributed along either north/south or east/west transects of the site. Scatterometer observations were made at angles of incidence and azimuth view angles equivalent to those for the SIR-B on any given orbit. The system output products were $\sigma^{0}$ as externally referenced to a Luneberg lens. Each $\sigma^{0}$ is the average of at least 100 independent samples depending upon angle of incidence. As a consequence, a statistically significant sample population was created for establishing the transfer function for each image which covered the area co-observed by the truck systems and SIR-B.

\subsection{SIR-B Antenna Patterns and Transmit Power}

For each of the six SIR-B data-takes over the site, the ARCs were used to record the time history of the incident power density. The output voltage of the ARC is proportional to the received power at the input to the ARC receiver. The transfer function of each ARC was established in the laboratory by injecting signals of pulse width $\tau=30.3 \mu \mathrm{s}$ and monitoring the output voltage as a function of input power $\mathrm{P}_{r}$ and pulse repetition frequency (PRF) from $1200 \mathrm{~Hz}$ to $2000 \mathrm{~Hz}$ :

$$
V_{\text {out }}=K(P R F) \times P_{r}
$$

where $K$ (PRF) is the ARC transfer coefficient. 


\subsection{Azimuth Patterns}

An example of the time-history of an ARC output voltage recorded during a SIR-B overpass on 11 October, 1984 is shown in Figure 2.

Depending on the level of the output power of the SIR-B transmitter, the ARC receivers generally recorded the second or third sidelobes within the linear portion of the receiver response. The average observed sidelobe levels are given in Table $2 \mathrm{as}-12.0 \mathrm{~dB}$ and $-10.3 \mathrm{~dB}$ for the lead and lag sidelobes (relative to STS attitude) respectively. The half-power ( $3 \mathrm{~dB}$ ) and null-to-null beamwidths $\beta$ are calculated from estimates of the STS velocity and range to target as derived from the preliminary ephemeris data. The average preliminary estimate of $\beta_{3 \mathrm{~dB}}$ is $1.1^{\circ}$. The variances around the calculated means are found to be very small.

The azimuth cuts recorded by the ARCs exhibit no evidence of pulse-to-pulse variation in SIR-B transmit power over their respective 3 to 4 second time histories ( $\approx 5,000$ pulses). All measured patterns are smooth and well behaved.

\subsection{Range Patterns}

The range antenna pattern assumed by JPL in the SIR-B digital correlator is shown in Figure 3. The research objective was to use the ARC measurements to verify this model for the antenna as deployed in orbit.

Since the range (elevation) pattern of the SIR-B antenna can not be measured directly from the observations made by a single ARC, it must be inferred from the observations of many receivers distributed in the range direction. The deployment of the ARCs with respect to any given shuttle orbit is depicted schematically in Figure 4. Typically, the ARCs were distributed over a $15 \mathrm{~km}$ to $20 \mathrm{~km}$ range extent which roughly corresponds 
to $5^{\circ}$ in angle depending upon the slant range for a particular orbit. As a consequence, it was possible to examine only a limited portion of the range pattern by forming a composite of the ARC measurements for a given orbital pass. Ideally, many more ARCs or other receivers should be distributed over a greater range extent in order to clearly def ine the shape of the pattern.

Because the transmit power from SIR-B varied with time (probably due to arcing in a coaxial cable), the differences in incident power density recorded at the ground by the ARCs were not solely related to the range antenna pattern. Hence, the ARC output voltage was used to estimate the product of the transmit power $P_{t}$ and the antenna gain $G_{t}$ by:

$$
P_{t} G_{t}=4 \pi R^{2} P_{r} / A_{e f f}
$$

where $\bar{R}$ is the siant range (as estimated at the stated angie of the antenna boresight relative to nadir using the preliminary ephemerides and assuming a spherical earth) and $A_{\text {eff }}$ is the effective apperture of the ARC (as measured for each ARC on an antenna range).

In Figure $5, P_{t} G_{t}$ is plotted for each ARC measurement as a function of the angular offset between the position of the ARC and the ground intersect of the antenna boresight as calculated from the preliminary ephemerides. The least-squares fitting of the assumed beam pattern (as shown in Figure 4) to the measured data yields the curves plotted in Figure 5. If it is assumed that the modeled range pattern is correct, then it is clear that: (1) there is a significant variance in SIR-B pass-to-pass transmit power as indicated by the peaks of the curves plotted in Figure 5 , and $(2)$ the estimates of the antenna boresight derived from the preliminary ephemerides can lead to as much as a $3^{0}$ error in the application of the antenna pattern to the radar data in the digital correlator. The angular offsets depicted in Figure 5 are belleved to be the 
result of two factors: (1) errors in the preliminary ephemerides and (2) discrepancies between the true antenna attitude as deployed in the shuttle bay and the shuttle attitude as measured in the nose of the shuttle. The first factor can be tested and may be correctable by using ephemerides based upon the shuttle "path tape" provided by the Johnson Space Center six months after the mission. The second factor is related to attitude and location dependent thermal loading on the shuttle which, at present, yields a noncorrectable error source with an uncertainty estimated to be \pm 10 .

Hence, while the true range pattern of the SIR-B antenna cannot be defined from the ARC measurements, it is shown that errors in the preliminary ephemerides can lead to substantial misapplication of the modeled antenna gain function in digital processing of the SIR-B data if the assumed model is correct. The expected magnitudes of these errors are shown graphically in Figure 5 and listed in Table 3. Conversely, the ARC data does not indicate that the assumed model for the range gain pattern is incorrect. By assuming the true boresight location errors to be as indicated in Table 3 for each orbit and assuming $G_{t}$ to be time constant (wherein only $P_{t}$ varies from pass-to-pass), then normalizing all ARC data with respect to the maximum $P_{t} G_{t}$ and boresight angle error for each pass yields the data composite shown in Figure 6. This approach, while certainly less than rigorous, indicates that the assumed beam form could be quite accurate.

\subsection{Transmit Power}

For the six orbits observed, the maximum $P_{t} G_{t}$ estimated from the ARCs varied from $85.1 \mathrm{dBm}$ to $88.0 \mathrm{dBm}$ (Table 3). Since it is reasonable to assume that $G_{t}$ was time-constant, $P_{t}$ was found to vary over a $3 \mathrm{~dB}$ range. Given a nominal transmit power of $1 \mathrm{KW}(60 \mathrm{dBm})$ and a maximum 
$G_{t}$ of $33.8 \mathrm{~dB}$ (from the antenna manufacturer and thermal vacuum tests),

the maximum $P_{t} G_{t}$ was expected to be $93.8 \mathrm{dBm}$ as compared to the average observed (of six passes) maximum $P_{t} G_{t}$ of $86.7 \mathrm{dBm}$. Hence, the average observed loss in the expected transmit power is estimated as 7.1 $\mathrm{dB}$ with a pass-to-pass standard deviation of $1.1 \mathrm{~dB}$.

The within-pass variability of SIR-B transmit power can be examined from plots of cross-track (range) averages of digital number as a function of time. For the Illinois data, these averages were found to be relatively constant yet they typically exhibited a short term fluctuation about the mean of \pm 0.4 to $0.5 \mathrm{~dB}$ and a period of about 0.25 to 0.33 seconds.

However, since the test site is a mixed agricultural scene with scattered towns, these variations may be scene related and not necessarily due to fiuctuations in SiR-8 transmit power. A sample is shown in Figure 7 for data-take 97.2. It is planned to iterate this analysis considering only scene elements for a single land-cover category (such as corn fields) once the SIR-B data is fully registered to extensive ancillary data pertaining to the along-track crop-type distribution. As mentioned previously, no random pulse-to-pulse variation in $P_{t}$ was recorded by the ARC receivers.

\subsection{SIR-B Image Calibration}

Many potential applications of orbital imaging radar involving quantitative parameter retrievals require strict standards of relative, if not absolute, radiometric calibration of the imagery. Effective methods of image calibration enable the comparison of data derived over time, between sites, and amongst suites of sensors using generalized algorithms. Considerable effort was taken to examine two external calibration methods to provide end-to-end transfer functions for the 
SIR-B imagery obtained over the Illinois test site. Targets of known radar cross-section were provided by the ARCs (as transponders) and by area-extended agricultural fields observed by two truck-mounted scatterometers.

Application of these methods to the SIR-B data was partially frustrated by the fact that many passes did not image the portion of the test site containing targets of known radar cross-section (Figure 1). However, the ascending data-takes (49.2 and 97.2) preceded by STS attitude alignment manuevers did image this area and consequently form the basis of the ensuing discussion.

\subsection{Image Transfer Function for SIR-B Digital Data}

The form of the transfer function for the conversion of SIR-B image digital number DN to relative units of radar backscattering coefficient $\sigma^{0}$ $\left(\mathrm{m}^{2} / \mathrm{m}^{2}\right)$ is completely defined by the SIR-B radar and the image formation processing.

To relate DN to $\sigma^{\circ}$, use will be made of large agricuiturai fieids that are represented by a large number of pixeis on the SAR image. Thus, for each field an average digital number, $\overline{D N}$, can be obtained by averaging over all the pixels of that field. This $\overline{\mathrm{DN}}$ value corresponds to the mean scattering coeffieicnt of the field $\sigma^{\circ}$, which is exactly what was measured by the truck scatterometers during the SIR-B mission; for each field, the scatterometers made several hundred measurements of the backscattered power in order to arrive at the mean backscattering coefficient $\sigma^{\circ}$.

For a given pixel i, the JPL processor produces a digital number $D_{\mathrm{i}}$ that is proportional to the square-root of the power $P_{j}$ received from the ground cell corresponding to the pixel $\mathfrak{i}$ on the processed SAR image, 


$$
D N_{i}=K P_{i}^{1 / 2}
$$

where $K$ is a scaling factor consisting of the receiver gain and an arbitrary image scaling coefficient. If the field under consideration consists of $M$ pixels and $P_{i}$ is a random variable with mean value $\bar{P}$, then for very large $M$

$$
\overline{\mathrm{DN}}=\left\langle K P_{i}^{1 / 2}\right\rangle=K\left\langle P_{i}^{1 / 2}\right\rangle=K\left\langle P_{i}\right\rangle^{1 / 2}=K \bar{P}^{1 / 2}
$$

where the order of the averaging and square-root operations have been reversed because the Central Limit Theorem is applicable ( $M$ is large). The power $P_{i}$ is given by

$$
P_{i}=\left(V_{s i}+V_{n i}\right)^{2}
$$

where $V_{s i}$ and $V_{n i}$ are the backscattered signal voltage and the receiver noise voltage, respectively, both of which are random variables. The voltage $\mathrm{V}_{\mathrm{si}}$ is a random variable because of scintillation (signal fading) resulting from the coherent addition of the signals backscattered from the scatterers in the ground cell corresponding to pixel $\underline{i}$. The voltage $V_{n i}$ is a random variable with a mean $\bar{V}_{n}=0$. The average value of $P_{i}$ is

$$
\bar{P}=\left\langle\left(V_{s i}+V_{n i}\right)^{2}\right\rangle=\left\langle V^{2}{ }_{s i}\right\rangle+2\left\langle V_{n i}\right\rangle\left\langle V_{s i}\right\rangle+\left\langle V^{2}{ }_{n i}\right\rangle=\bar{P}_{s}+\bar{P}_{n}
$$


where $\bar{P}_{S}$ and $\bar{P}_{n}$ are the mean signal and noise powers. Furthermore,

$$
\bar{P}_{s}=\frac{\bar{P}_{t} G^{2}{ }_{\theta} \lambda^{2} \sigma \sigma_{\theta} A_{\theta}}{(4 \pi)^{3} R^{4}}=c_{\theta} \sigma_{\theta}{ }_{\theta} A_{\theta}
$$

where $P_{t}$ is the transmitted power; $G_{\theta}, A_{\theta}$, and $R_{\theta}$, are the antenna gain, resolution cell area and range to target, respectively, at incidence angle $\theta$; and $\lambda$ is the wavelength. The constant $C_{\theta}$ represents the factors multiplying $\sigma_{\theta}^{\circ} \mathrm{A}_{\theta}$.

Combining Eqs. 4, 6, and 7, gives

$$
\overline{D N}=K\left[C_{\theta} \sigma_{\theta}^{0} A_{\theta}+\bar{P}_{n}\right]^{1 / 2}
$$

Solving for $\sigma_{\theta}^{\circ}$ leads to

$$
\sigma_{\theta}^{\circ}=\left(\overline{D N}^{2}-K^{2} \bar{P}_{n}\right) / C_{\theta} A_{\theta} k^{2}
$$

In $\mathrm{dB}$,

$$
\sigma_{\theta}^{O}(\mathrm{~dB})=10 \log \left(\overline{\mathrm{DN}}^{2}-\alpha\right)-\beta
$$

where $\alpha=K^{2} \bar{P}_{n}$ and $\beta=10 \log \left(C_{\theta} A_{\theta} K^{2}\right)$. 
For each SIR-B digital image, the scaling factor $K$ is given by the product of the receiver gain and the image scaling coefficient; $A_{\theta}$ is readily calculated; $C_{\theta}$ is a modeled function of $P_{t}, G_{\theta}$, and $R_{\theta}$; and $\bar{P}_{n}$ can be approximated from the DN obtained for an area expected to be in the noise (i.e., the DN from a specular surface such as a smooth water body). Ideally, all coefficients in Eq. 9 are known. In practice, $C_{\theta}$ is imperfectly known due, primarily, to uncertainty in $P_{t}$ and $G_{\theta}$.

\subsection{Area-Extended Targets}

In the ensuing preliminary analysis, only the SIR-B data from the ascending orbits will be considered. This data was reprocessedusing the post-mission JSC path-tape to provide the ephemerides which should leave only small residual errors related to the antenna boresight position and negligible ranging errors. For each agricultural field observed by the truck-mounted scatterometers, a mean digital number was computed as the average of 80 to 120 pixels which excluded boundary pixels and point target responses. The scatterometers operated at angles of incidence and azimuth view angles identical to that for a given SIR-B pass, and produced mean $\sigma^{\circ}$ from $\approx 100$ independent samples per field.

Each data pair (i.e., mean SIR-B DN and mean $\sigma^{\circ}$ ), was used to provide an independent estimate of the amalgamated transfer coefficients via Eq. 10. The noise power $\bar{P}_{n}$ is determined from small water bodies. The resultant variance in the $\beta$ transfer coefficients as calculated for each discrete area-extended target should be related to : (1) fading in the radar cross-sections measured by either the truck-mounted scatterometers or the SIR-B target response. (2) errors in the antenna gain function assumed 
in the digital processor, and/or (3) fluctuations in the SIR-B transmit power.

The effects of fading are reduced by the averaging of relatively large sample sizes. The $2 \sigma$ uncertainty due to fading is calculated to be $\leq 0.5$ $\mathrm{dB}$ in both cases. The test fields were distributed over a distance of about $20 \mathrm{~km}$ in both range and azimuth. Over this distance, Figure 7 shows a one $\sigma$ uncertainty in range-averaged $P_{t}$ of $\leq 0.5 \mathrm{~dB}$. In addition to the two preceding random processes, errors in the application of the antenna gain pattern in the image processing should yield a range dependent bias in the calculated transfer coefficients.

The calibration results from data-take 97.2 are plotted in Figure 8 . The average transfer coefficient $\beta$ determined from 40 observations is $47.86 \mathrm{~dB}$ with a standard deviation of $0.85 \mathrm{~dB}$. No distinctive trends are observed in $\$$ as a function of either range or azimiuth iucatiuns uf the area-extended targets.

\subsection{Point Targets}

A calibration approach similar to the preceding was used for the SIR-B response to point targets. The SAR data had been processed to produce 4-look images to reduce image speckle. When a point target of radar cross-section $\sigma_{\text {ARC }}$ is present in a homogeneous ground cell of area $A_{\theta}$ and average backscattering coefficient $\sigma_{b}^{0}$, the received signal voltage, in the absence of fading, is

$$
v_{S}=c_{\theta}\left(\sigma_{D}^{\circ} A_{\theta}+\sigma_{A R C}\right)^{1 / 2}
$$


The background backscattering coefficients $\sigma^{\circ}{ }_{b}$ (of the six cells within which the six ARCs were later deployed) were measured by the truck-mounted scatterometers and found to vary from -17 to $-15 \mathrm{~dB}$ at $\theta=30^{\circ}$ (which is the antenna elevation angle for orbits 49.2 and 97.2). The ARCs had radar cross-sections (RCSs) between 25 and $28 \mathrm{dBm}^{2}$, and $A_{\theta}=28.9 \mathrm{dBm}^{2}$. Thus, the background contribution $\sigma_{b} A_{\theta}$ is smaller than the ARC contribution by about 12 to $16 \mathrm{~dB}$. For a four-look average, a $14 \mathrm{~dB}$ ratio of target RCS to background RCS implies a measurement standard derivation of $+1.6 \mathrm{~dB}$ and $-1.9 \mathrm{~dB}$ due to signal fading variations of the background [2]. These error limits could have been improved by using less attenuation in the ARCs and thereby increasing $\sigma_{\mathrm{ARC}}: \sigma_{\mathrm{b}}$. For example, $\sigma_{\mathrm{ARC}}: \sigma_{\mathrm{b}}=20 \mathrm{~dB}$ yields a fading uncertainty of about $\pm 1 \mathrm{~dB}$.

Upon using Eq. 11 to define $V_{5}$, the resultant expression for DN is

$$
D N=k\left[c_{\theta}\left(\sigma_{b} A_{\theta}+\sigma_{A R C}\right)+\bar{P}_{n}\right]^{1 / 2}
$$

When considered alone for data-take 97.2, the ARCs yield an average estimate of the transfer coefficient $\beta=47.94 \mathrm{~dB}$ with a $1 \sigma$ deviation of $1.34 \mathrm{~dB}$. This deviation is within the error bounds related to fading of the background contribution. In terms of the mean $\beta$ coefficients, the point targets alone agree with the area-extended targets to within $0.1 \mathrm{~dB}$. The average transfer function of the two approaches is plotted in Figure 8 for comparison with the data. The larger standard deviation about the mean for the point target calibration results can be attributed to the small sample size (i.e., number of ARCs) and to the effects of fading.

In order to check the accuracy of the combined transfer function for data-take 97.2, $\sigma^{0}$ SIR-B was calculated from the mean DN for each target 
and regressed versus the $\sigma^{0}$ measured by the truck-mounted scatterometers.

$$
\sigma^{0} \operatorname{SIR-B}(d B)=10 \log \left(\overline{D N}^{2}-144\right)-47.9
$$

The uniform distribution of the scatter in Figure 9 indicates that the form of the transfer function is appropriate. In addition, the scatter is shown to be within the $95 \%$ confidence interval related to fading.

\subsection{Between Pass Stability}

The SIR-B transfer function derived for the other ascending pass over the Illinois site, data-take 49.2 , is shown in Figuro 8 for comparative purposes. The between-pass difference of the average transfer coefficients $\beta$ was found to be $2.7 \mathrm{~dB}$. This between-pass difference is approximately that estimated by insertion of all known or modeled quantities into Eq. 10 for the two passes with the assumption that there is a $2.4 \mathrm{~dB}$ difference in the SIR-B transmit power as recorded by the receivers (Table 3). This strongly suggests that the SIR-B was stable over time except for the uncommanded fluctuations in the transmit power.

4.0 Discussion

Observations at the Illinois test site showed the SIR-B antenna to be fully illuminated and to produce a nominal azimuth antenna pattern. Analysis of the same data with respect to the range pattern is not conclusive, but indicates that the range antenna pattern assumed in processing may be accurate assuming that the boresight location can only 
be estimated to within $2^{0}$ from the preliminary ephemeris data. The SIR-B transmit power was found to be $\approx 7 \mathrm{~dB}$ below specifications and exhibit a pass-to-pass variability of $\approx 3 \mathrm{~dB}$. Pulse-to-Pulse variation of $\mathrm{P}_{\mathrm{t}}$ was not observed. Observed fluctuations in range-averaged digital number $\left( \pm 0.5 \mathrm{~dB}\right.$ ) cannot be unequivocably ascribed to variance in $P_{t}$ and may be caused by the true scene variance.

In spite of variance in the SIR-B transmit power, end-to-end transfer functions generated from either point targets or area-extended targets were found to yield close agreement (within $0.1 \mathrm{~dB}$ ) for a given pass. Variance in the transfer coefficients established by either method were found to be within the confidence intervals expected from fading considerations. Pass-to-pass changes in the transfer coefficients agree with observed differences in SIR-B transmit power.

In view of these findings, it is concluded that targets of known cross-section can be readily and effectively used to provide transfer functions for orbital SAR. The use of area-extended targets for this purpose yields the most satisfactory results. However, the use of point targets is far simpler and may be the preferred alternative for many situations. In either case, it is important to make use of more than one target in order to reduce uncertainties related to fading, to target deployment, or to measurement errors of the radar cross-section of the target. In addition, the siting of point targets and their cross-section relative to that of the background must be carefully considered in order to optimize performance. Finally, due to uncertainty in the projected swath location for shuttle-based missions, calibration targets should be broadly distributed in the range direction to ensure inclusion of several targets within the image swath. 
References

[1] Brunfeldt, D.R. and F.T. Ulaby, "Active Reflector for Radar Calibration", IEEE Trans. Geoscience Remote Sensing, vol. GE-22, no. 2, 1984.

[2] Ulaby, F.T., R.K. Moore, and A.K. Fung, Microwave Remote Sensing: Active and Passive, Vol. Il: Radar Remote Sensing and Surface Scattering and Emission Theory. Reading, MA: Addison-Wesley, 1982.

Acknowledgements

This work was supported by the Jet Propulsion Laboratory under Contracts 956921 and 957191 as subcontracts under NASA Contract NA57-918. 


\section{LIST OF TABLES}

1. Data-takes over the Illinois test site.

2. Azimuth antenna pattern summary.

3. Estimates of maximum $P_{t} G_{t}$ and preliminary boresight errors from receiver measurements.

\section{FIGURE CAPTIONS}

1. Map of SIR-B coverage. Each swath is annotated with its respective data-take number.

2. Typical azimuth beam-form observed by an ARC.

3. Assumed model of SIR-B range antenna pattern used in processor.

4. Sketch of active radar calibrator ARC deployment.

5. $P_{t} G_{t}$ as observed by the ARCs for each data-take. Angular of fset is calculated from estimates of boresight location based upon preliminary ephemerides.

6. ARC measurements normalized to the antenna beam-form as depicted in Figure 5 for each data-take.

7. Range-averaged digital number as a function of azimuth location for data-take 97.2. The mean $\mathrm{DN}=92.13 \pm 5.42$.

8. SIR-B transfer functions for data-takes 49.2 and 97.2

9. Regression of $\sigma^{0}$ SIR-B as calculated from mean DN via Eq. 13 with $\sigma^{\circ}$ as measured for each target. 


\begin{tabular}{|c|c|c|c|}
\hline Date & Data-take & $\begin{array}{c}\text { Look } \\
\text { Direction }\end{array}$ & $\begin{array}{l}\text { Local Angle } \\
\text { of Incidence }\end{array}$ \\
\hline $10 / 07 / 84$ & 38.1 & NE & 17 \\
\hline \multirow[t]{2}{*}{$10 / 08 / 84$} & * 49.2 & NW & 30 \\
\hline & 54.1 & NE & 38 \\
\hline $10 / 09 / 84$ & 70.1 & NE & 50 \\
\hline $10 / 10 / 84$ & 86.1 & NE & 59 \\
\hline $10 / 11 / 84$ & *97.2 & SW & 31 \\
\hline • & & & \\
\hline
\end{tabular}




$\begin{array}{llc}\text { Mean } & \begin{array}{l}\text { Standard } \\ \text { Deviation }\end{array} & \begin{array}{c}\text { N of } \\ \text { Samples }\end{array}\end{array}$

Beamwidth:

$3 \mathrm{~dB}$

Null to Null

$1.095^{\circ}$

$2.421^{\circ}$

$.038^{\circ}$

$.049^{\circ}$
26

20

Sidelobe Level:
Lead
$-11.96 \mathrm{~dB}$
$0.73 \mathrm{~dB}$
20
Lag
$-10.34 \mathrm{~dB}$
$0.74 \mathrm{~dB}$
21

Values are based upon preliminary ephermeris data. 


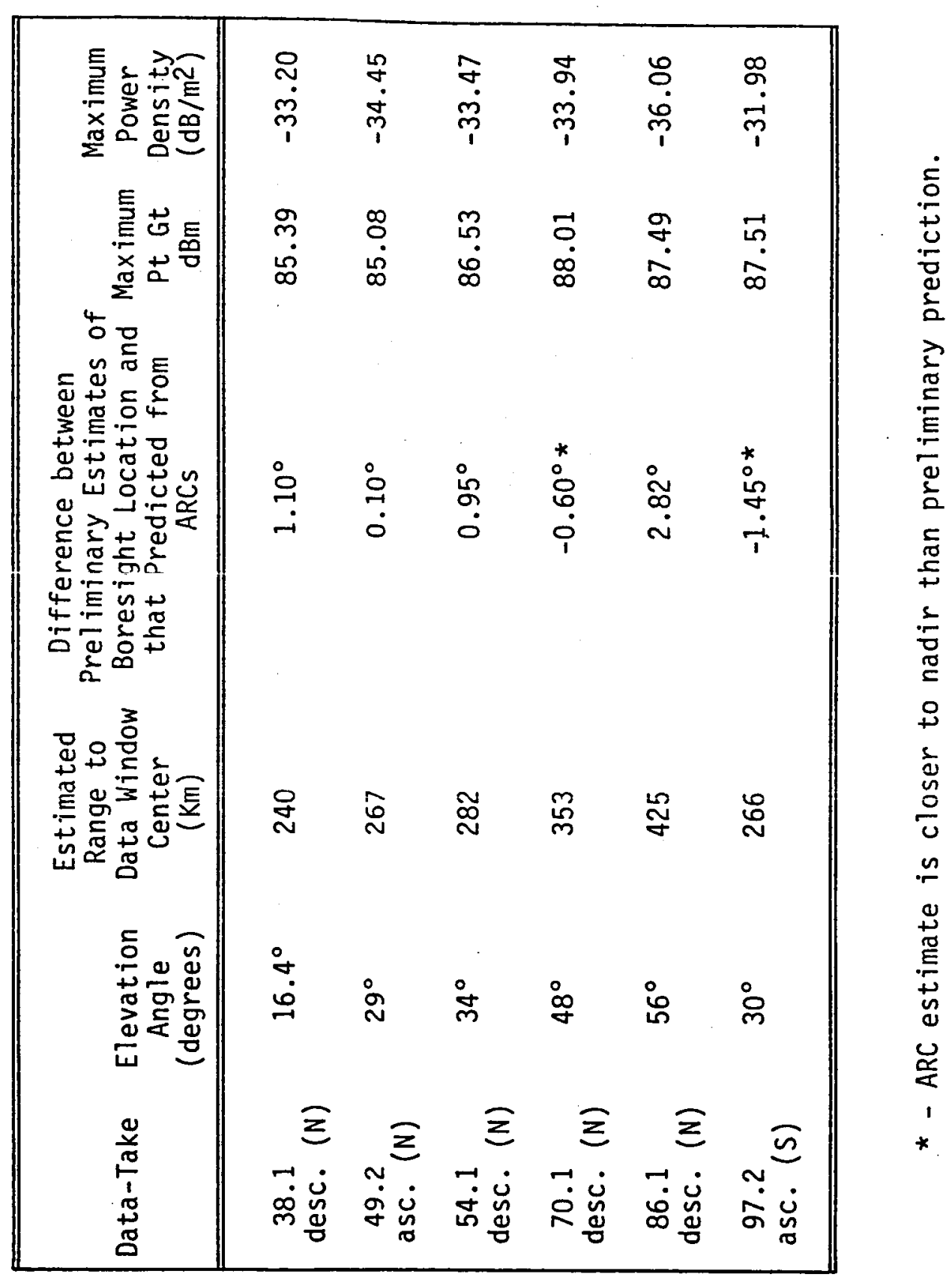




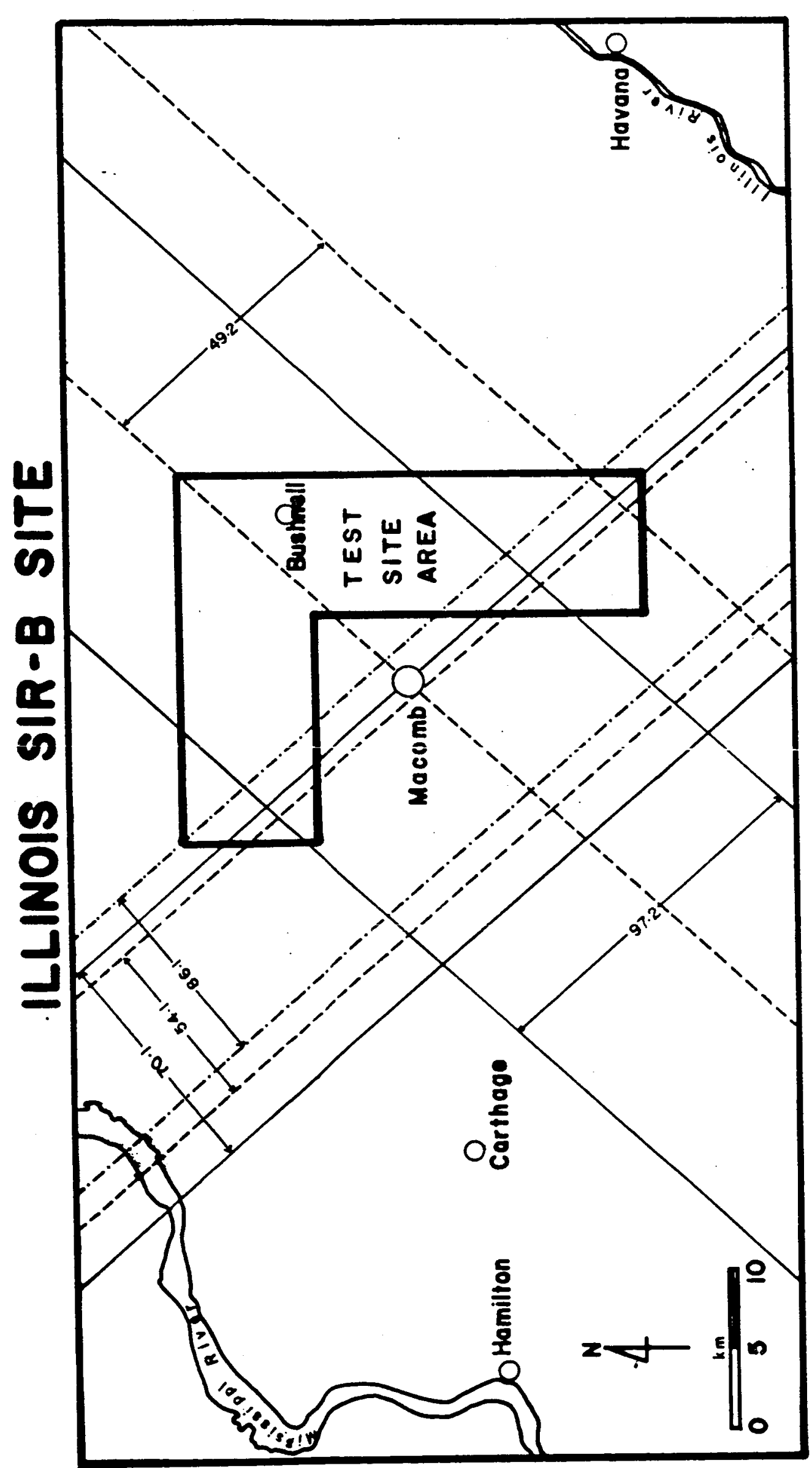




\section{SIR-B AZIMUTH PATTERN}

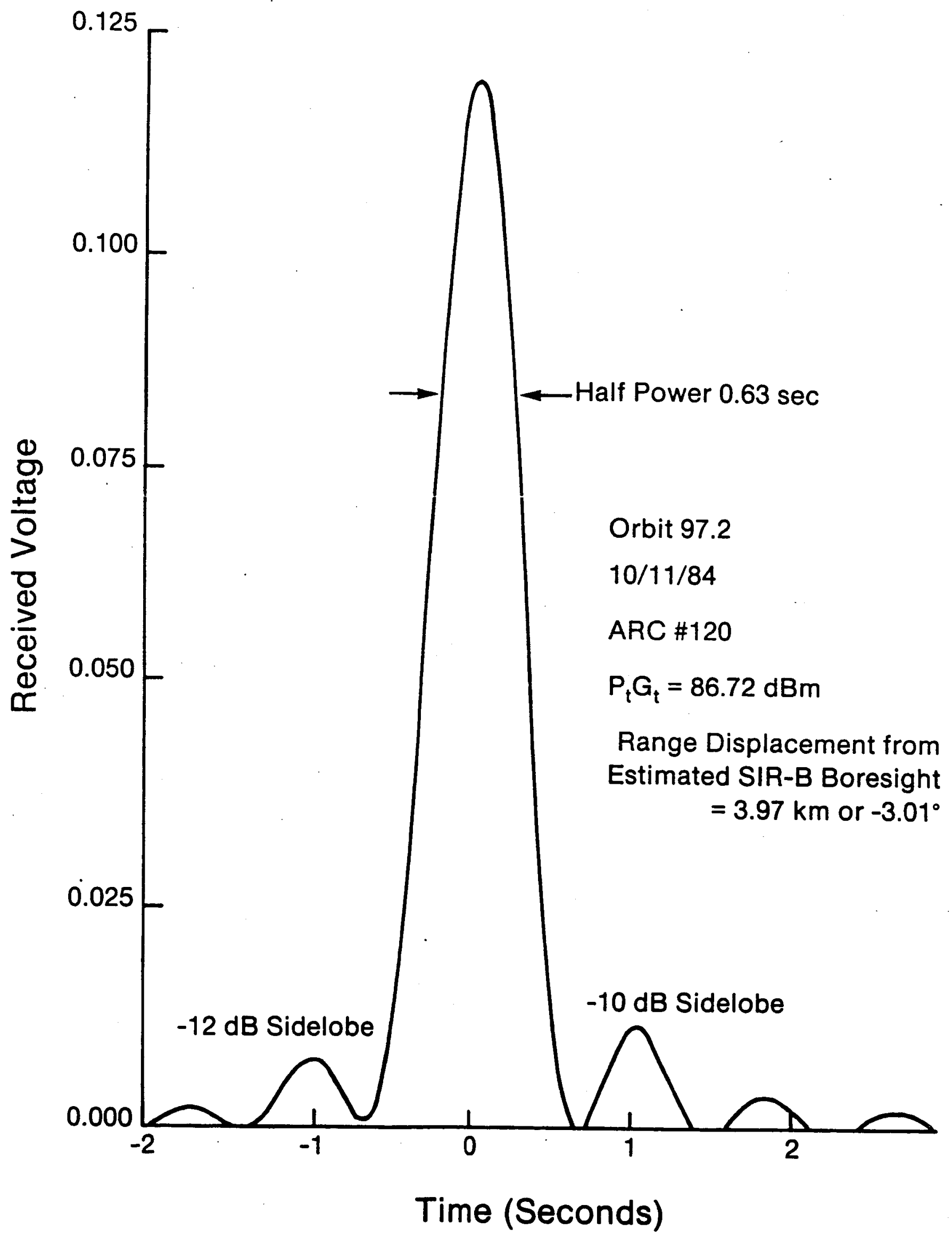




\section{ASSUMED SIR-B RANGE PATTERN}

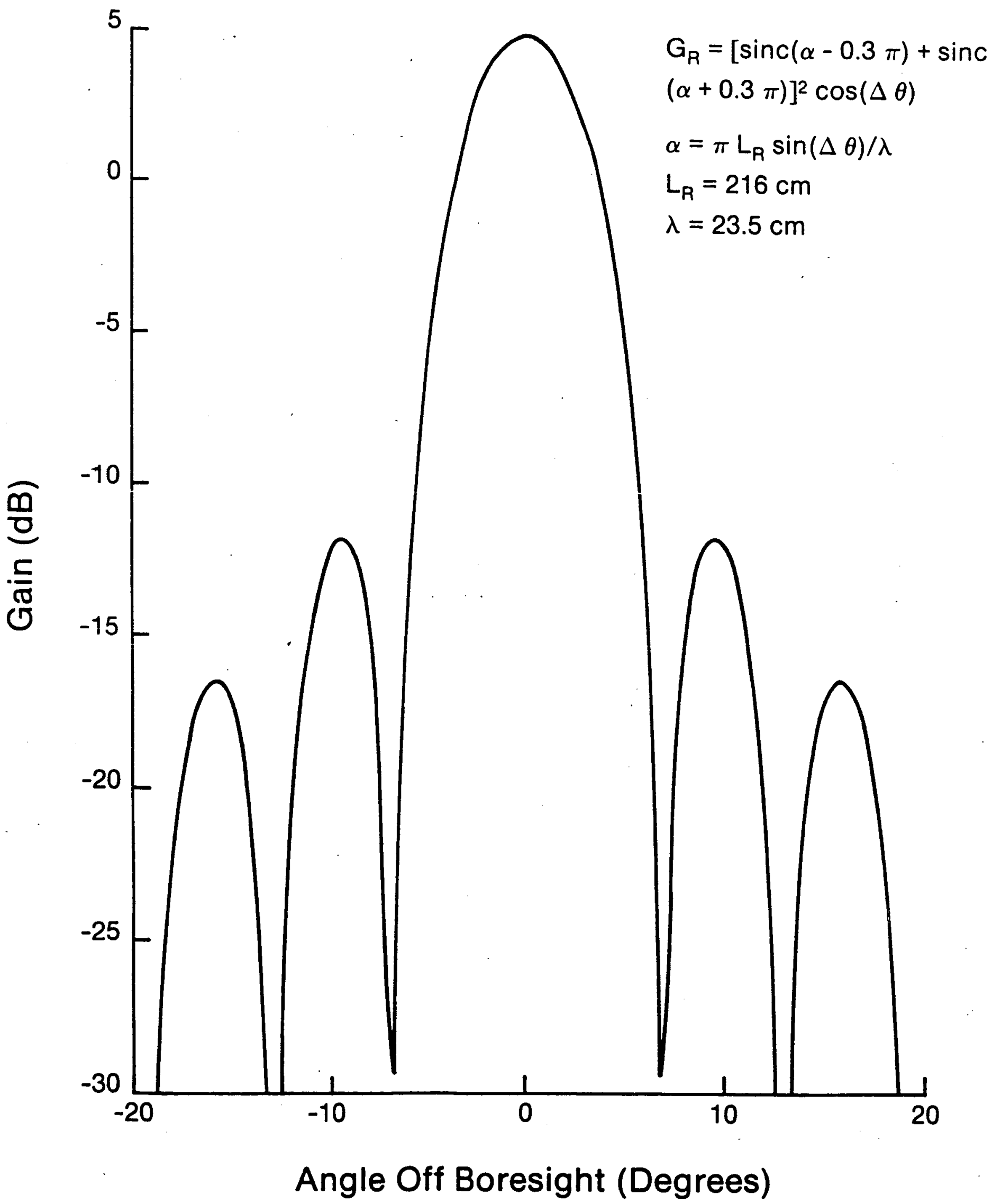




\section{SIR-B ANTENNA PATTERNS FROM ARCs}

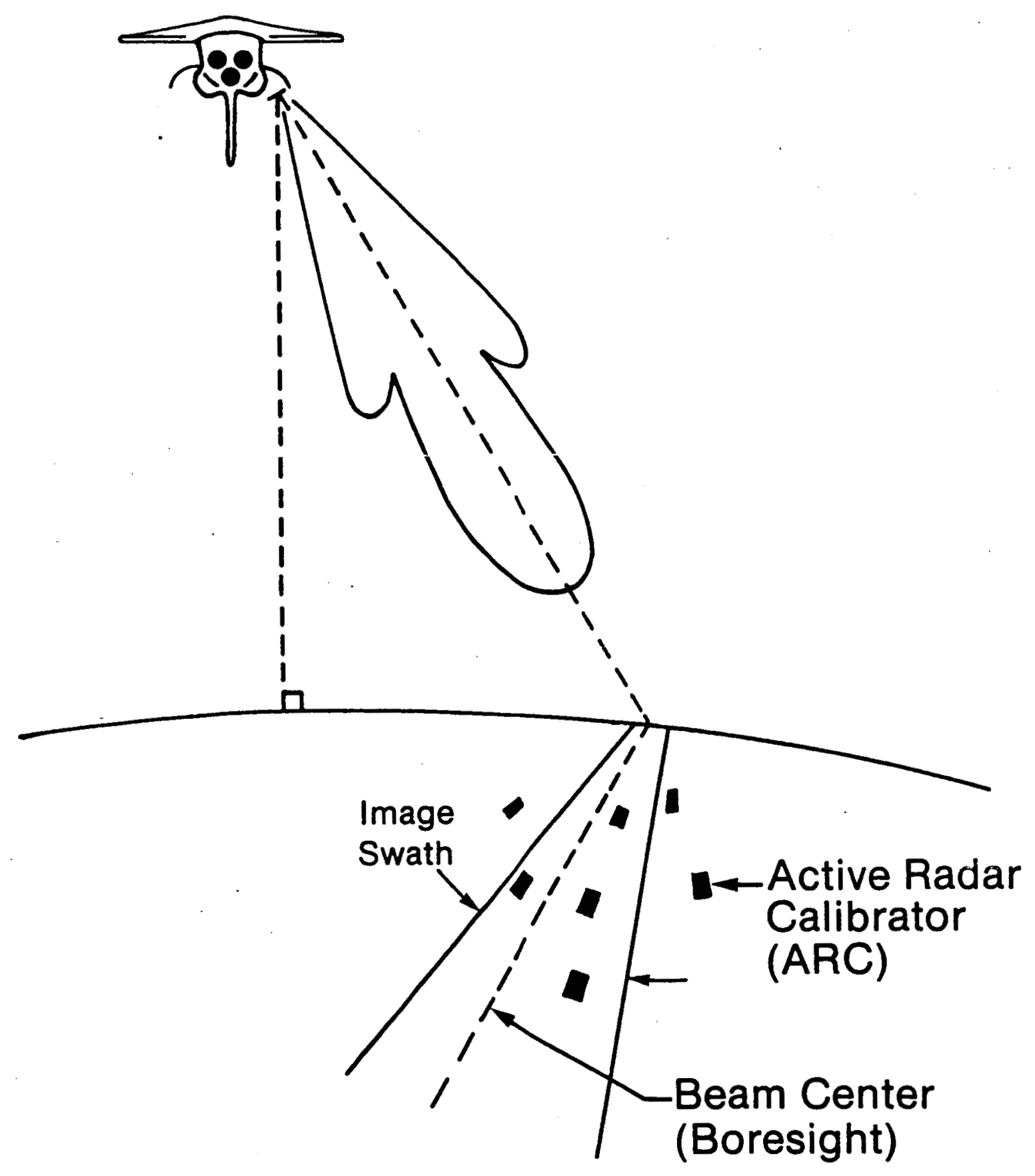




\section{DIFFERENCE IN BORESIGHT POSITION AS MEASURED BY ARCS AND ASSUMED FROM PRELIMINARY EPHEMERIS}
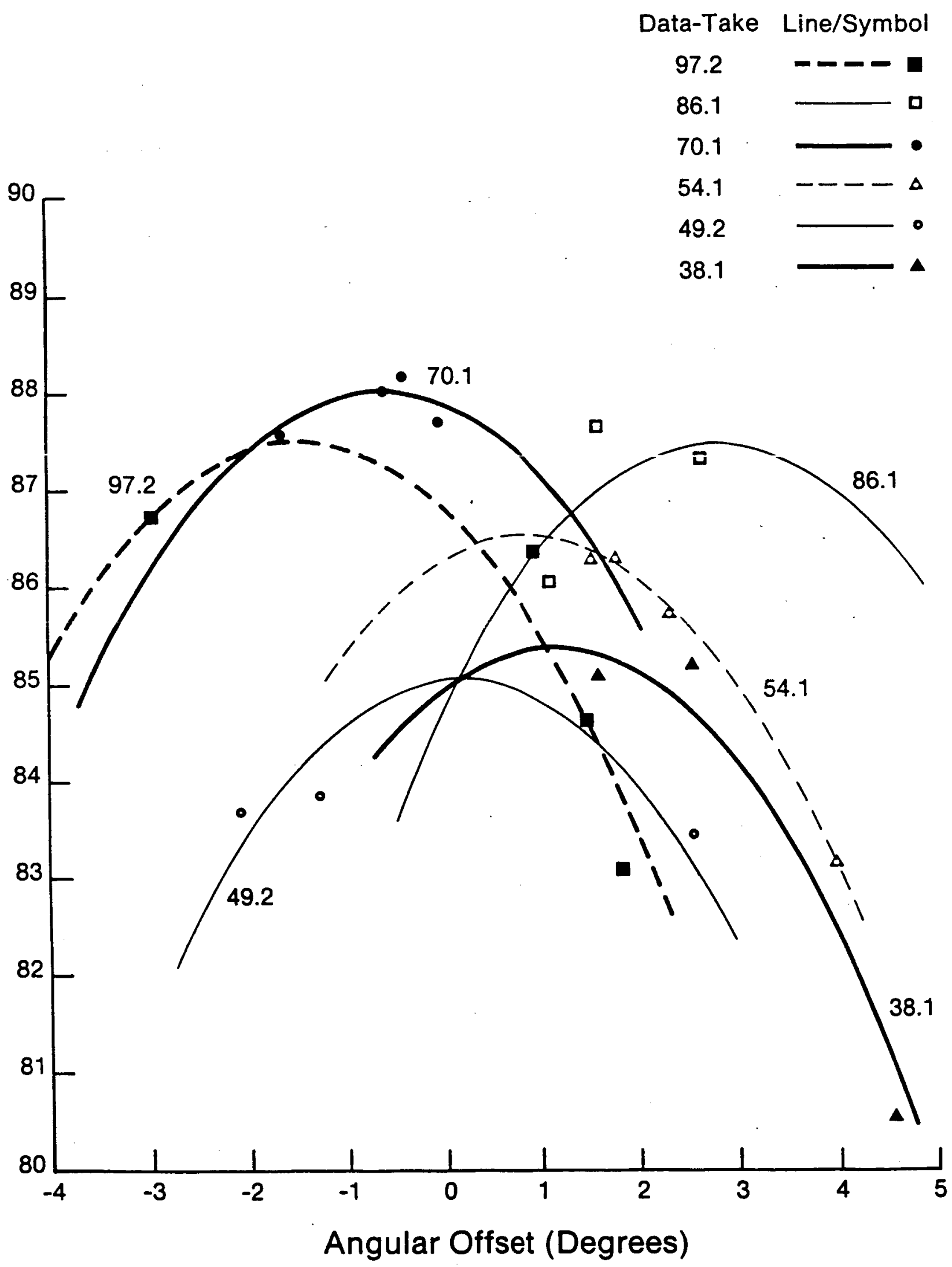


\section{ARC MEASUREMENTS RELATIVE TO RANGE PATTERN}

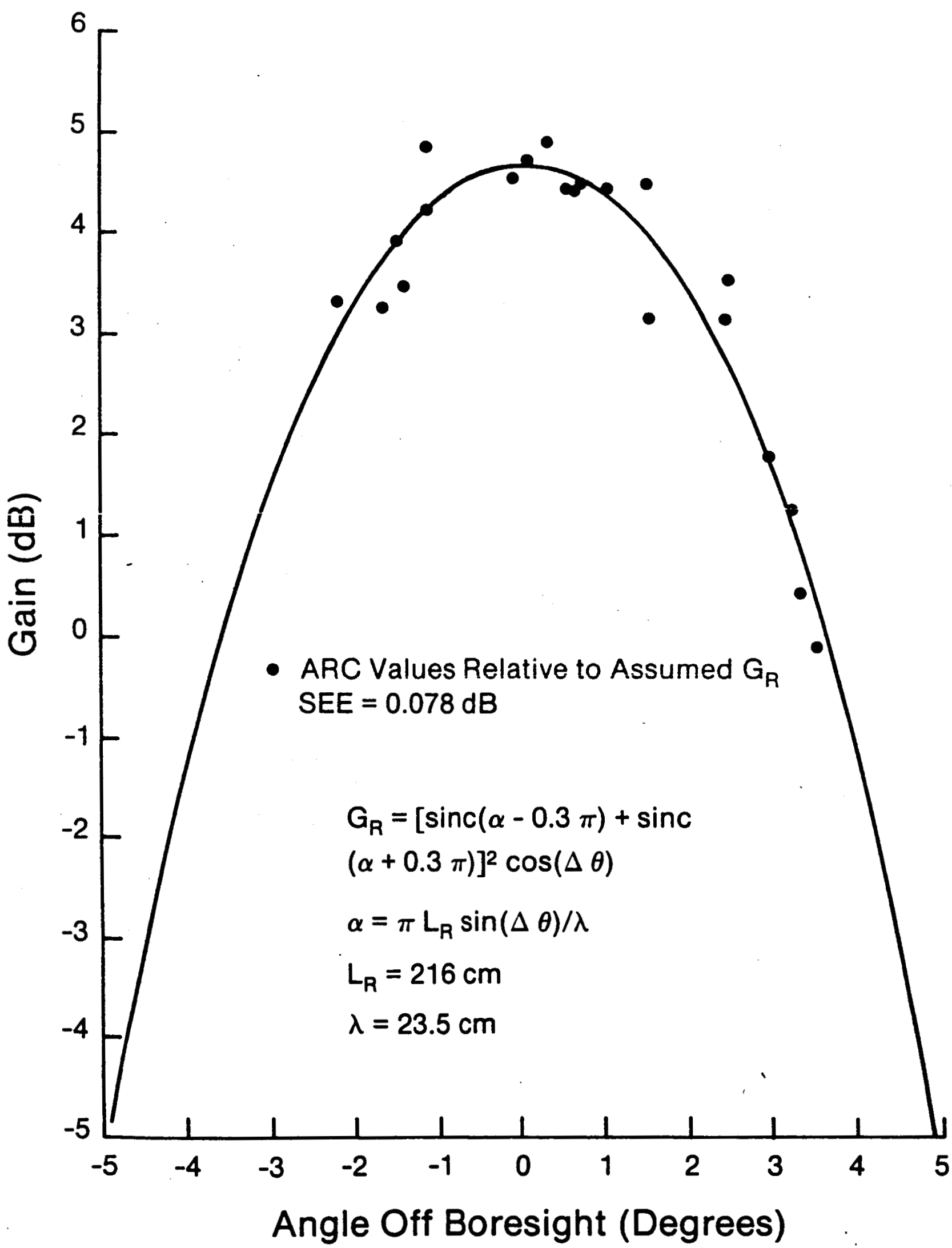




\section{Average Digital Number}

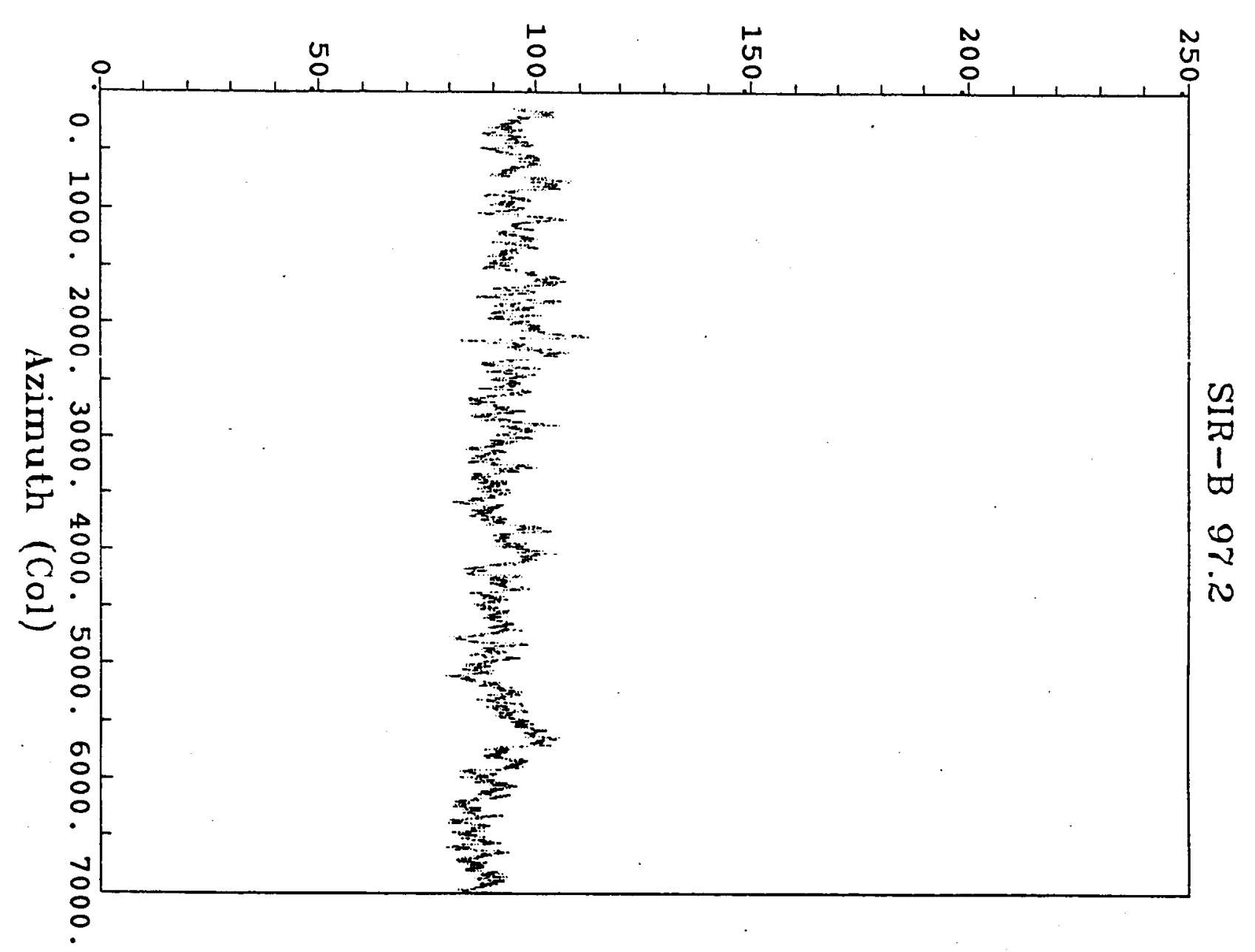


CALIBRATION ACCURACY FOR DATA-TAKE 97.2

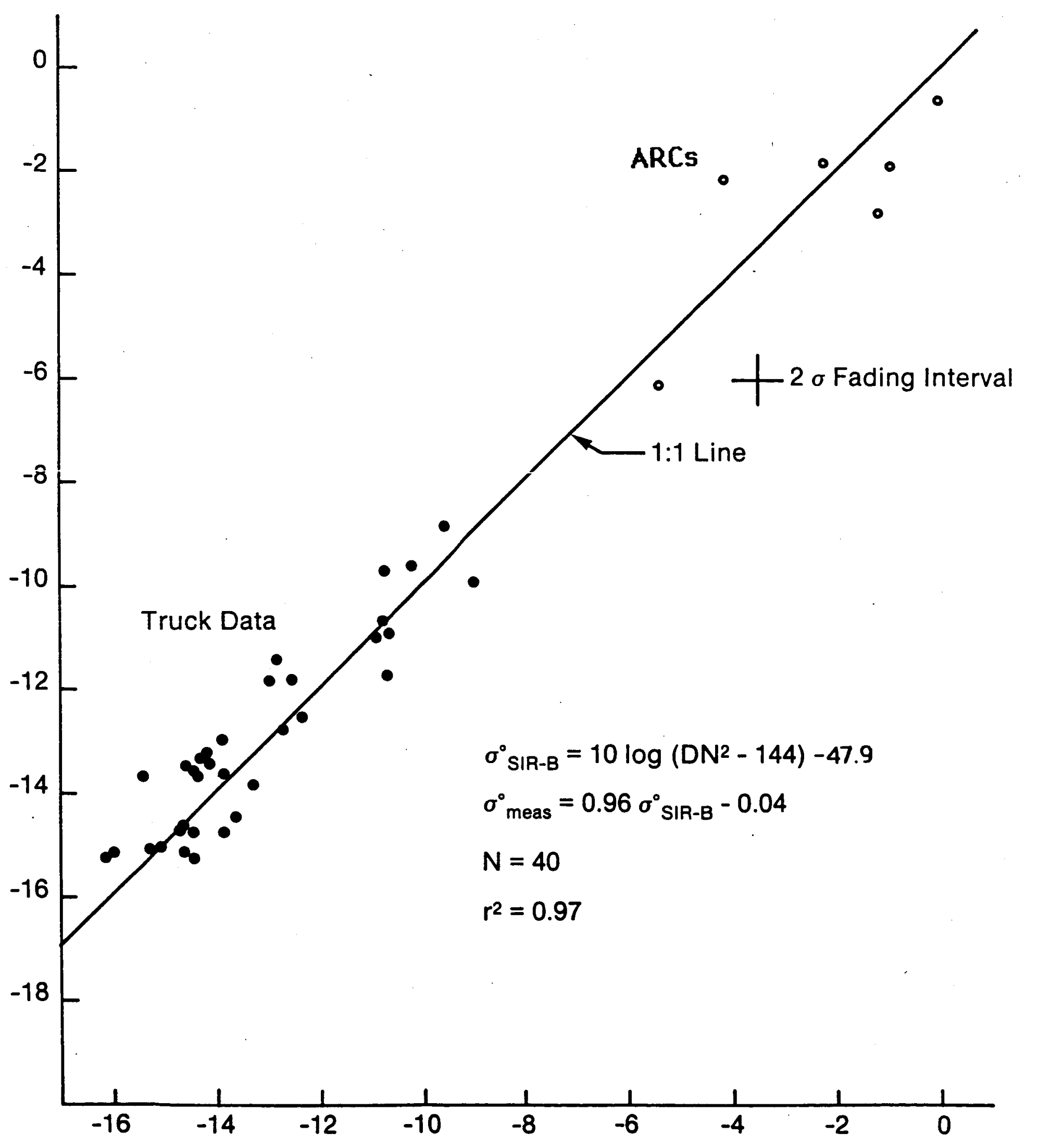

Calculated $\sigma_{\text {SIR-B }}^{\circ}(\mathrm{dB})$ 